\title{
Esthétique, architecture et environnement montagnard. Nouveaux
} outils, nouveaux symboles

Monique Barrué-Pastor, Michel Barrué

\section{Citer ce document / Cite this document :}

Barrué-Pastor Monique, Barrué Michel. Esthétique, architecture et environnement montagnard. Nouveaux outils, nouveaux symboles. In: Études rurales, n¹17, 1990. Architecture rurale : questions d'esthétique. pp. 103-117;

doi : 10.3406/rural.1990.4652

http://www.persee.fr/doc/rural_0014-2182_1990_num_117_1_4652

Document généré le 31/05/2016 


\begin{abstract}
Aesthetics, architecture and the mountain environment : new tools, new symbols

This attempt to reassess the relations between architecture, society and environment has come out of the construction of new buildings for livestock in a touristic zone protected under the French law on historical monuments. In this experimental research action project, the decision to link theory and practice (and thus break the narrow relationship usually established between aesthetics, nature and nostalgia) had to do with the idea that modernity does not necessarily break with the past. Two notions that shaped this analysis (integrated local development and architecture's aesthetic dimension) were defined by their capacity for taking into account a place, society and history. As operational concepts, they could be used to link "development" with "protection" so as to avoid the "conservationist" approach, which often shapes issues and perceptions of the mountain environment.
\end{abstract}

\title{
Résumé
}

Une tentative de réévaluation des rapports entre architecture, société et environnement est faite à partir de la construction de nouveaux bâtiments d'élevage dans une zone touristique protégée au titre des Monuments historiques, dans le contexte expérimental d'une recherche-action. La volonté d'articuler théorie et pratique en rompant avec le rapport étroit trop souvent établi entre esthétique, nature et nostalgie s'accompagne de l'idée que la modernité n'est pas forcément en rupture avec le passé. Deux notions structurent l'analyse, le "développement local intégré" et la "dimension esthétique" d'une architecture, et sont définies par leur capacité d'intégration dans un lieu, dans une société, dans une histoire. Transformées en concepts opératoires, elles ont permis de coordonner l'option de "développement" et celle de "protection", en évitant l'approche "conservatoire" qui prime très souvent dans la diversité des enjeux, des usages et des formes de perception de l'environnement montagnard. 
MONIQUE BARRUÉ-PASTOR E'T MICHEL BARRUÉ

\author{
Esthétique, architecture \\ et environnement montagnard
}

Nouveaux outils, nouveaux symboles

Nous analyserons ici un processus de production architecturale à partir d'un exemple de conception et de réalisation de bâtiments d'élevage en site classé. Cette expérience de "recherche-action" 1 nous a permis d'élaborer la notion de développement local intégré, et nous a amenés à définir la dimension esthétique d'une architecture par sa capacité d'intégration dans un lieu, dans une société et dans une histoire.

Les hautes vallées du Luchonnais, dans les Pyrénées, fournissent un exemple typique du cumul des contraintes et des atouts qui caractérisent actuellement les zones de mont agne. Exclusivement consacrée à l'élevage, cette région a connu depuis 1860 et jusqu'aux années 1970 un mouvement continu de régression de ses activités et de sa population. La situation s'est alors stabilisée. Le recensement de 1982 enregistre même une légère progression avec l'installation de nouveaux habitants. Des activités se développent, liées au tourisme (d'été et d'hiver) et au thermalisme, au moment même où le mouvement de déprise agricole et de mise en friche, avec les méfaits qu'il entraîne (déséquilibres écologiques, catastrophes, incendies, etc.), commence à imposer l'idée que le maintien

1. Programme de recherche "Bâtiments d'élevage et installation de jeunes agriculteurs en montagne pyrénéenne", 1983-1987. Financement : CEE-AGRIMED, MRT, DMDR ; responsables : $M$. Barrué-Pastor (CIMA-CNRS, Institut de Géographie - Université de Toulouse II) et $M$. Barrué (École d'Architecture de Toulouse). 
d'un minimum d'activité pastorale et de vie sociale permanente est nécessaire, notamment pour la protection des paysages.

Or, sous les effets de la restructuration économique et de l'extension du chômage, un nombre croissant de jeunes décident de s'installer et de reprendre une exploitation familiale, mais avec des objectifs de vie et de travail bien différents de ceux de leurs prédécesseurs. Ces jeunes exploitants sont porteurs de dynamisme et d'ouverture, mais aussi de changement dans les pratiques agricoles, et par contrecoup dans les paysages. Pour développer l'élevage, la construction de bâtiments neufs est devenue primordiale. Dans cette zone, en effet, la grande majorité des bâtiments datent du XIXe siècle et ne sont plus adaptés aux besoins actuels. Par ailleurs, la plupart de ces jeunes exploitants pratiquent une activité saisonnière liée au tourisme. La multiplication des échanges avec les citadins a modifié en partie leurs référents culturels et les a sensibilisés à la protection des paysages, "leurs" paysages, reconnus d'intérêt national, érigés en patrimoine naturel prestigieux, et qui sont leur dernière richesse monnayable. C'est pourquoi le problème de l' "intégration paysagère" des nouveaux bâtiments agricoles a été posé d'emblée, la plupart des constructions neuves se trouvant en sites “classés" ou "protégés" au titre de monuments historiques. D'autre part, la transplantation de bâtiments industriels conçus sur les modèles de la plaine a été jusqu'ici un échec. Ils se sont avérés inadaptés aux besoins, aux moyens et aux contraintes de ces éleveurs montagnards. Sortir de l'impasse était urgent, il fallait trouver des références nouvelles, aussi bien méthodologiques que techniques, architecturales ou paysagères, pour répondre à la complexité des enjeux. Notre recherche s'inscrit ainsi dans une réévaluation des rapports entre architecture, société et environnement.

\section{Les politiques de développement et de protection, leurs enjeux esthétiques}

La construction de nouveaux bâtiments d'élevage, dans une zone touristique protégée au titre des monuments historiques, pose inévitablement en termes de choix esthétiques le problème de la diversité des usages et des formes de perception de l'environnement naturel. Des enjeux économiques, symboliques, idéologiques, sociaux et culturels se trouvent mêlés dans un processus complexe de changement, fait de transitions mais aussi de ruptures. D'où la difficile coordination, pour ne pas dire l'opposition, entre l'option du "développement" et celle de la "protection". La loi montagne (1985), qui tentait de conjuguer les deux impératifs, ne permet pas de surmonter le dilemme [Barrué-Pastor 1987 : 217-237]. 
Selon J.-C. Chamboredon [1985: 140], "l'avènement de la nature comme pure nature n'est pas dissociable d'une histoire sociale et économique de la campagne et d'une histoire de la relation ville/campagne". Or, si l'évolution des formes de consommation du cadre naturel est majoritairement marquée par le passage de l'appropriation utilitaire à la jouissance "désintéressée", on ne peut plus réduire les choses à une simple opposition entre une société rurale dont l'utilitarisme serait tout entier rivé à une logique agro-pastorale et une société urbaine au regard de laquelle l'espace montagnard ne serait qu'un espace de loisir et de conservation. Sous les effets du tourisme, pratiques sociales et usages de l'espace se sont diversifiés selon des objectifs parfois contradictoires. Il faut abandonner les schémas déterministes qui liaient tel système de représentation à tel groupe social et admettre l'existence de relations beaucoup plus nuancées où intervient la diversité des pratiques et des enjeux sociaux. Certains agriculteurs sont sensibles aux thèses "conservatoires" de logiques de développement touristique dans lesquelles ils sont désormais engagés. Certains citadins ont à l'inverse épousé la théorie d'une défense de l'environnement passant par le maintien et le développement d'une activité agricole qui mobilise les moyens techniques de son temps (à l'inverse d'une conception traitant celle-ci en relique folklorique, susceptible d'entretenir le pittoresque montagnard). Il n'en existe pas moins deux modes différents de rapport à la nature, présents simultanément dans ces zones de montagne. Dans le premier mode, la campagne est devenue nature. Lieu de repos, de régénération, de contemplation, elle est associée à une certaine idée de virginité dont la traduction esthétique extrême est l'absence de toute trace récente de l'action de l'homme. Cette fonction symbolique de la campagne comme nature s'est d'autant plus développée qu'à la déprise agricole correspondent des paysages de moins en moins marqués par le travail des hommes.

La politique qui s'inspire de cette idéologie vise à transformer la campagne en un cadre idyllique pour le développement de nouveaux usages non productifs, dominés par la fonction de loisir. Elle repose sur l'objectif de conservation d'un patrimoine devenu "paysager" dont serait éliminée, au nom de l'esthétique, toute trace d'activité agricole moderne. Le développement touristique, en revanche, aurait la capacité de protéger la qualité de ce patrimoine naturel et culturel. Nous retrouvons là des options communes à certains écologues et à certains architectes des Bâtiments de France. Elles impliquent le refus de voir l'agriculture se transformer en fonction des impératifs de la modernité, de la rentabilité, de l'amélioration des conditions de vie et de travail. La violation de la nature correspond à celle de l'"ordre éternel des champs". Est ici en cause le degré d'acceptation de l'humanisation, et des formes de celle-ci. Il varie avec les goûts esthétiques, dont l'histoire ne peut être 
séparée de celle des perceptions sociales et des systèmes de domination. L'esthétique traduit un certain état des rapports entre société et nature et les normes de goût et de valeur ne sont pas dissociées des normes du savoir : "Les grilles de lecture du paysage utilisées par l'esthète ou le savant se superposent sans brouiller les regards. Les normes de l'émotion recoupent celles de l'intelligibilité." [Briffaud 1989 : 21] Les arguments esthétiques et scientifiques d'une certaine élite se sont longtemps épaulés pour imposer l'hégémonie d'une position protectionniste.

Il semble que nous vivons actuellement un renouvellement des vieilles valeurs du XVIIIe siècle, par le biais d'une réévaluation de l'ancienne esthétique pittoresque, qui a donné naissance au nouveau paysan “jardinier de la nature”. La préoccupation esthétique (ou plutôt sa déviation esthétisante) devient le support de nouvelles formes de revalorisation et de réinsertion de zones déclarées jusqu'ici "marginales", et qui sont en train d'acquérir un nouveau statut. Il s'agit de trouver des formules alternatives de développement et de gestion des ressources, basées sur la sauvegarde de ce qui est devenu patrimoine régional ou national, au détriment d'une vie sociale permanente et équilibrée. L'esthétisme est une nouvelle forme d'intégration à l'économie de marché des zones marginales (des zones de montagne entre autres), où les paysans ont perdu leur fonction productive.

A l'opposé, une autre politique de développement est possible, correspondant à une autre conception de la nature, mais aussi à la réalité des mutations actuelles de la population rurale. Après une phase d'éclatement et d'antagonisme entre les différents usages du territoire, en effet, commence une nouvelle phase de recomposition culturelle, idéologique et politique. Il faut voir là sans doute une conséquence de la recomposition des sociétés villageoises, mais aussi l'effet d'une résistance nouvelle de l'agriculture, résistance qui passe par un processus de réappropriation et de marquage du territoire. D'où le caractère symbolique que revêt la construction de nouveaux bâtiments. Ceux-ci doivent traduire un ensemble d'attentes où sont étroitement mêlés le moderne, le fonctionnel, le durable, et aussi le beau. Ils doivent répondre à la fois aux impératifs de protection des paysages et à ceux $d u$ développement agricole. Le concept d'intégration acquiert dans ce contexte une place centrale, qu'il s'agisse de “développement local intégré" ou $d^{\prime}$ "architecture intégrée".

\section{Une nouvelle problématique de l'intégration architecturale}

Maintenir sur place la population en améliorant ses conditions de vie, d'une part, préserver le paysage, d'autre part : au lieu d'opposer ces deux objectifs, nous nous sommes efforcés, au cours de l'expérience de 
recherche-action que nous avons menée, de les poursuivre conjointement, et de les concilier. La démarche architecturale a eu pour but de trouver un modèle alternatif et les solutions techniques adaptées, en tenant compte des recherches qui, depuis les années 1970, essaient de faire évoluer les conceptions du développement, c'est-à-dire en s'appuyant sur les concepts d'intégration, de complexité, de transition, d'adaptation, de diversité et de spécificité, et en mobilisant les différentes catégories d'acteurs impliqués. Il nous a donc fallu développer une approche de l"intérieur" susceptible de rompre avec les modèles "extérieurs", construits au préalable et appliqués tels quels dans n'importe quel milieu. L'adaptation aux besoins et aux moyens disponibles suppose une modification de l'attitude des chercheurs impliqués dans une démarche où tous les choix doivent être justifiés et définis en fonction des individus directement concernés ; mais aussi une modification du comportement des architectes à l'égard du procès de construction. La place et le rôle de l'architecte dans notre société évoluent. Ce n'est certainement pas un hasard si cette expérience se situe à un moment où les architectes sont remis en cause. En quête d'une nouvelle légitimité mais aussi de nouveaux débouchés, ils sont amenés à s'investir dans de nouveaux domaines, notamment les lieux de travail et de production, abandonnés jusqu'ici aux techniciens et aux ingénieurs. Précisons qu'en harmonie avec les courants architecturaux qui se préoccupent de l'insertion du bâti dans son milieu social et naturel, la conception de bâtiments d'élevage en haute montagne a été ici considérée comme une démarche architecturale à part entière et non comme une production annexe, mineure.

\section{L'intégration des contraintes}

Le programme a été défini par les jeunes éleveurs en phase d'installation dans le Luchonnais, avec l'aide de techniciens agricoles et de l'équipe de recherche associant un architecte et un géographe.

Dès le départ, l'objet de la recherche a été défini comme un objet complexe, produit d'interrelations multiples entre des impératifs issus de domaines aussi divers que les systèmes techniques d'élevage, les contraintes topo-climatiques, les logiques familiales, les conditions de travail, le génie civil, les limites financières, les ressources locales, la protection des sites, paysages et monuments historiques. Impératifs qui, dans ces zones de montagne, ont pris très vite la forme de contraintes particulièrement fortes et dont l'analyse a prouvé que seule une approche globale intégrant l'ensemble des données et des connaissances disponibles pouvait résoudre le problème. La définition du programme de construction pourrait être résumée ainsi : ces bâtiments devaient être adaptés aux nouveaux modes d'exploitation agricole en 
montagne, résistants et fermés, en raison des conditions climatiques, tout en étant peu coûteux, évolutifs et intégrés au paysage, étant donné la forte pression touristique locale et les exigences de la protection des sites.

L'intégration paysagère étant un impératif majeur, la prise en compte de la dimension esthétique est essentielle à la maîtrise des rapports entre architecture, société, économie, culture et environnement naturel, à l'opposé d'une conception en termes de luxe ou de supplément d'âme. La qualité esthétique ne peut être en aucun cas un facteur d'augmentation des coûts. Nous avons essayé de montrer qu'elle pouvait être un facteur de l'intégration architecturale dans son contexte historique et dans son environnement ${ }^{2}$.

\section{L'intégration des disciplines}

Le choix théorique de la "complexité" suppose la coopération entre sciences sociales, sciences naturelles et techniques. C'est ainsi que le noyau initial (architecture et géographie sociale) s'est élargi aux domaines de l'agronomie, de la zootechnie, de l'écologie, de la sociologie et de l'ergonomie. Par ailleurs, le processus de construction, dans ses méandres administratifs et financiers, implique de nombreux partenaires. $\mathrm{La}$ synergie des compétences, des pratiques et des savoir-faire a permis de dépasser les cloisonnements institutionnels et les conflits liés aux impératifs sectoriels, et peut-être d'éviter que le compromis nécessaire ne se réduise au plus petit dénominateur commun... Elle a permis aussi, et surtout, de donner à l'architecte une possibilité d'agir en milieu rural autrement qu'en intervenant parachuté, incompréhensible et incompris, ou, à l'opposé, en censeur, comme c'est le cas pour l'architecte des Bâtiments de France, et d'éviter que l'intégration paysagère ne se limite à l'esthétique de l'objet bâti ${ }^{3}$.

Cependant, cette multiplication des connaissances scientifiques et techniques ne pouvait être féconde que si elle dépassait le stade de l'expérimentation pour déboucher sur l'action. A ce jour, cinq grangesétables, deux granges-bergeries et une chèvrerie ont été construites sur les principes de cette démarche.

2. "C'est un trait caractéristique de la création actuelle que ses véritables fondateurs se rattachent à des terroirs bien précis; ils ne sont pas coupés de leurs racines..." [Giedion 1978, I : 29].

3. "L'architecte a tendance à référer ses jugements esthétiques à des essences fixes, de type objectif, comme les formes, cependant que le populaire opère suivant une esthétique à deux paliers : celle de l'habiter dans laquelle vérité et utilité s'accordent pour converger dans le convenable." [Segaud $1980: 25$ ] Cette remarque situe bien l'importance de l'articulation entre les architectes et les sociologues pour garantir la prise en compte du vécu et du non vu tout au long du processus de conception et de réalisation. 


\section{L'intégration de l'histoire}

Le refus de l'opposition entre passé et présent, opposition qui conduit inévitablement soit à des démarches "passéistes", le plus souvent imposées par tout l'arsenal juridique de la protection des sites et des paysages, soit, à l'opposé, à des solutions tout aussi extrémistes de fuite en avant, préconisées au nom d'une certaine vision réductrice du progrès, est certainement l'élément jouant le rôle le plus déterminant dans le choix de la problématique de l'intégration architecturale. Que signifie concevoir aujourd'hui une architecture par rapport à une histoire et à un lieu ? Que veut dire "intégrer" un bâtiment dans un site, en l'occurrence un pays montagnard très fortement marqué par l'organisation spatiale et architecturale d'une société rurale en pleine mutation? Est-il possible de dépasser la tendance actuelle à figer les réponses, soit selon les seuls critères esthétiques dominés par la vision conservatrice et passéiste, soit selon les seuls critères d'un fonctionnalisme moderniste, plaqué sur un milieu (naturel et social) inadapté et porteur de déstructuration ? Concevoir une architecture à la fois ouverte sur l'avenir et le progrès tout en étant enracinée dans l'histoire et la culture locales entraîne nécessairement la rencontre voire la fusion d'éléments issus du domaine de l'architecture "savante", des avancées les plus récentes des connaissances scientifiques (en agronomie, zootechnique, résistance des matériaux, etc.) et des enseignements de l'architecture vernaculaire.

Parmi les quatre manières de prendre en compte l'histoire dans la forme architecturale : l'imitation ou le mimétisme, la citation ou le collage d'éléments empruntés au passé, la métaphore, la réappropriation de l'architecture populaire et vernaculaire, c'est évidemment à la dernière qu'a été donnée la préférence. Cette réappropriation de l'habitat ancien passait par l'introduction d'innovations. En définitive, il s'agit de transgresser la référence pour la remettre en jeu. Cette démarche qui essaie d'allier patrimoine et innovation illustre cette réflexion de Georges Braque [1948: 85]: “L'avenir est la projection du passé conditionné par le présent." Elle vise aussi à démontrer que, pour avoir quelques chances de dépasser le stade expérimental, "les conceptions 'intégrées' du développement et de la gestion de la nature doivent s'enraciner historiquement pour mieux s'inscrire socialement". [Picon $1990: 7$ ]

\section{Un exemple d'architecture intégrée}

La conception, selon ces principes, des bâtiments d'élevage peut être analysée à plusieurs niveaux : le rapport entre volume, organisation spatiale et fonction; le rapport entre système constructif, matériaux et 
savoir-faire ; le rapport entre implantation, lieu, site et environnement. A chaque niveau fonctionne la double dialectique entre nature et culture, symbole et fonction.

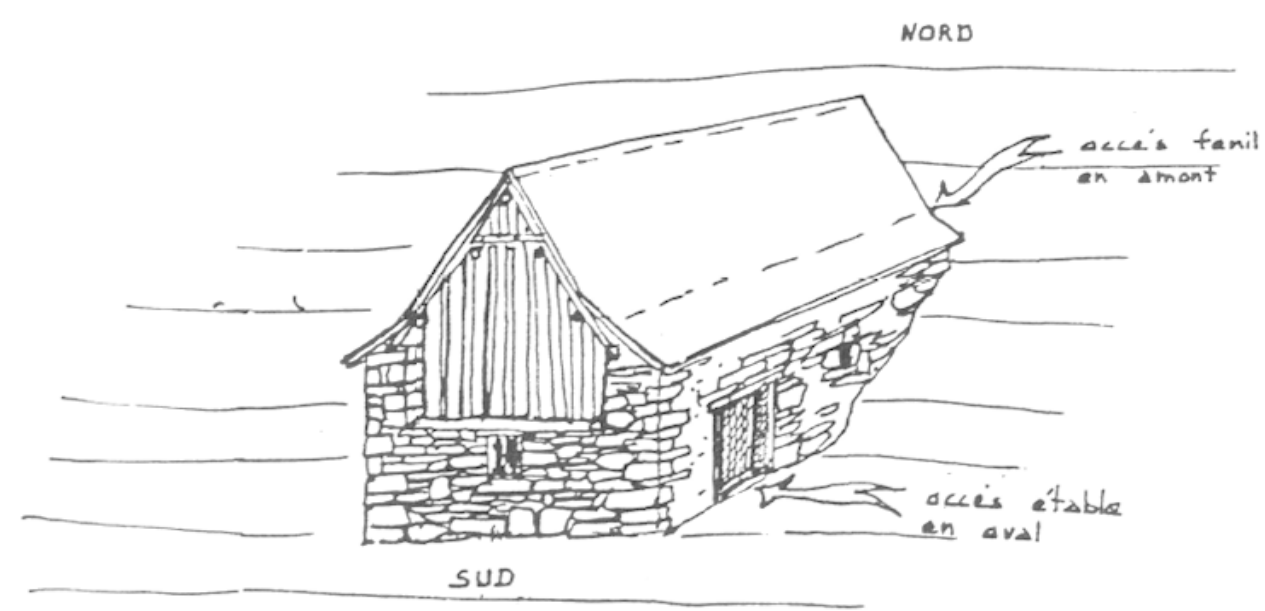

Fig. 1 - La grange luchonnaise. Implantation, organisation.

\section{Volume, organisation spatiale, fonction}

Le principe volumétrique qui a été retenu est en partie conforme aux caractéristiques du bâti ancien, dans la mesure où il regroupe sous un même toit les éléments qui définissent les bâtiments d'élevage : le stockage de l'alimentation et le logement des animaux. Ce choix semble en effet le mieux adapté à la vie hivernale et à l'exiguité des parcelles. En revanche, l'ampleur et la nature des nouveaux programmes ont entraîné des choix de dimension et d'organisation spatiale intérieure en rupture avec les principes des anciens bâtiments. Ces derniers se caractérisent en effet par de petits volumes à un étage, conçus pour loger et nourrir une dizaine de têtes de gros bétail (au maximum). Ils sont encastrés dans la pente perpendiculairement aux courbes de niveaux (cf. fig. 1) sur les versants sud (les "soulanes"). Cette organisation permet d'accéder directement, en aval, à "l'écurie" ${ }^{4}$ située au rez-de-chaussée et, en amont, au fenil situé à l'étage. La superposition du fenil et du logement des animaux (étable ou bergerie), séparant le sec et le propre de l'humide et du sale, correspondait aux modes de vie et de travail de la société agro-pastorale du siècle dernier.

L'organisation de l'espace intérieur proposé aujourd'hui a été déterminée par l'importance des troupeaux à loger (40 têtes de gros

4. Terme local pour signifier de manière indifférenciée le logement des animaux (équins, bovins, ovins, etc.). 
bétail ou 300 brebis en moyenne) et par l'existence de nouveaux systèmes de stockage et de distribution. Il s'agit d'un volume très important regroupant au même niveau l'alimentation et les animaux (cf. fig. 2 et fig. 3$)^{5}$.

Le volume central est réservé au stockage de l'alimentation nécessaire pour les cinq à six mois d'hiver. Il peut s'adapter à l'accueil de balles rondes de fourrage, de silos à ensilage et même de foin entré en vrac; il est flanqué de part et d'autre de deux appentis réservés au logement des bêtes. Ce parti architectural facilite une gestion rationnelle des tâches et notamment de la distribution de l'alimentation. Fn limitant les distances et les circulations, il réduit la durée et la "pénibilité" du travail de l'éleveur, qui est maintenant le plus souvent seul pour une charge dix fois plus importante qu'auparavant.

Le corps central, dont la toiture à forte pente (80\%) génère un grand volume, est adapté à l'importance de l'alimentation à stocker. Les appentis, dont la pente de toiture est plus faible (35\%), sont plus adaptés au logement du bétail. La variation de volume ainsi créée par le changement de pente de la toiture permet une diversité d'utilisation sans contredire l'unicité volumétrique. Ce choix architectural a par ailleurs l'avantage de faciliter le passage de l'ancien système au nouveau, en permettant une grande souplesse d'aménagement intérieur et d'utilisation des espaces. Il a permis dans certains cas la réintroduction partielle d'un plancher (reprenant en cela les formes anciennes du bâti, chères aux éleveurs) sans pour autant remettre en cause la cohérence du projet. A l'opposé, il a facilité l'intégration du séchage solaire des fourrages entrés en vrac dans le grand volume central, les toitures orientées au sud et couvertes en bac acier de couleur sombre, rappelant l'ardoise, servant de capteur solaire.

L'existence d'un grand volume central peut également, de manière paradoxale, faciliter un grand fractionnement des espaces intérieurs tout en garantissant la facilité de circulation. Cette possibilité répond aux besoins d'une chèvrerie-fromagerie. Un dernier exemple des diverses adaptations de ce principe unique nous est fourni par un bâtiment implanté à 1300 mètres (l'altitude la plus élevée) dont l'appentis amont a été supprimé en raison de la forte déclivité du terrain.

\section{Changement de pente et architecture vernaculaire}

Le changement de pente entre le volume central et les appentis a été traité en référence aux coyaux des toitures anciennes. Le coyau est un élément des charpentes traditionnelles à forte pente ; c'est une pièce de

5. Les nouveaux bâtiments, compte tenu de leur longueur, n'ont pu être encastrés dans la pente comme les anciennes granges. Leur implantation, parallèle aux courbes de niveaux, induit une démarche tout à fait nouvelle d'insertion dans le site. 


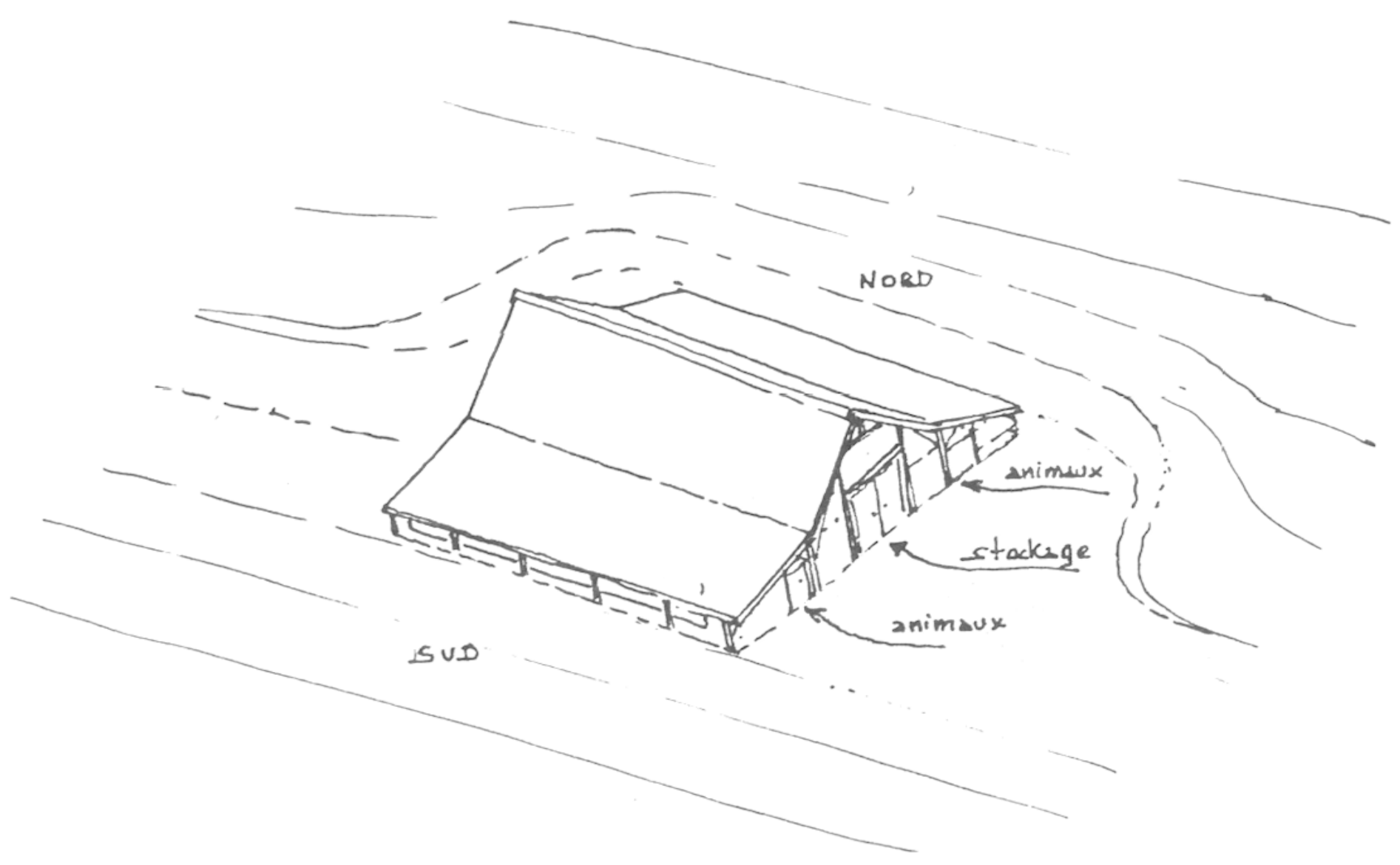

Fig. 2 - Les nouveaux bâtiments d'élevage. Implantation, organisation.

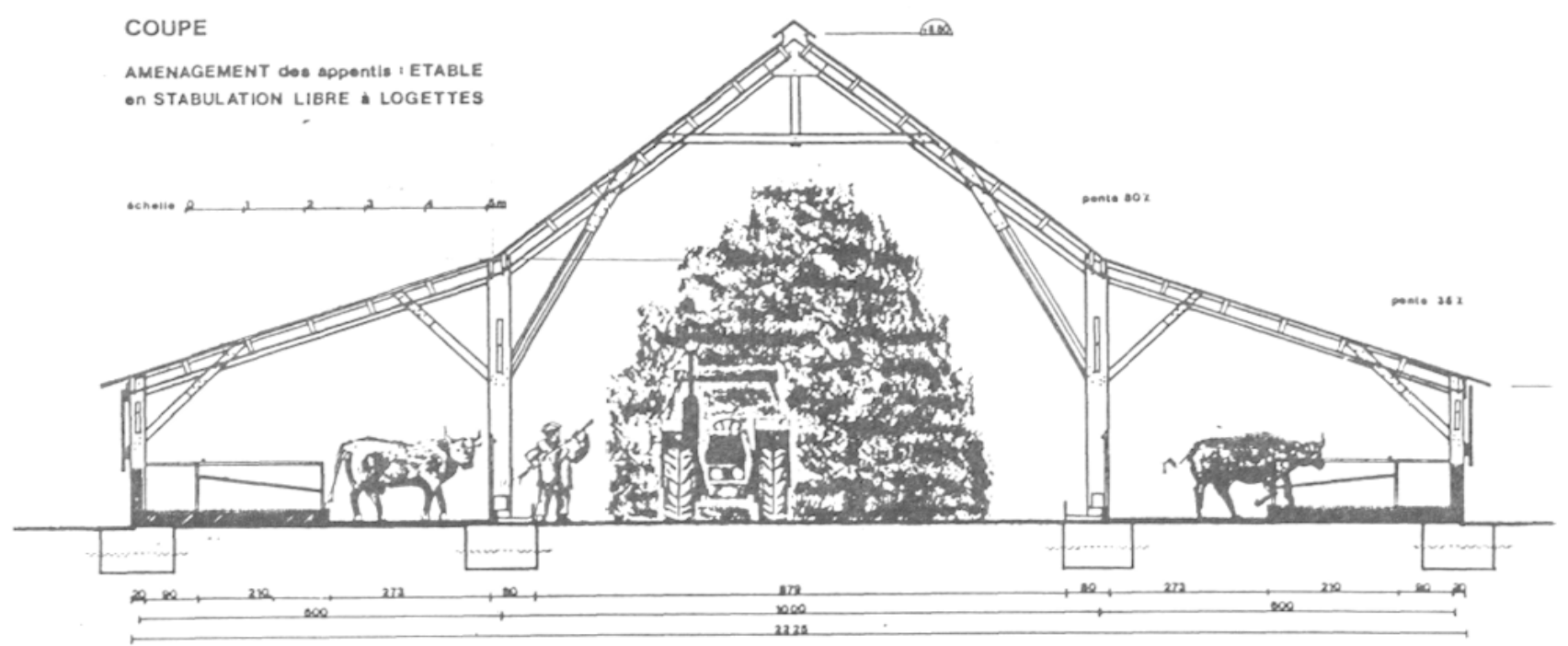

Fig. 3 - Organisation intérieure des bâtiments. 
bois qui prolonge le chevron au-delà du mur porteur et permet de le recouvrir, ce qui crée une inflexion de la partie inférieure de la toiture (cf. fig. 4). Le principe est d'autant plus intéressant que la pliure ainsi obtenue protège le mur gouttereau et renforce la charpente en établissant une continuité de toiture. Tout risque d'infiltration et d'arrachement par le vent est ainsi évité. Cet élément architectonique de l'habitat vernaculaire présente ainsi toutes les qualités esthétiques et techniques d'adaptation au site et au climat montagnards.

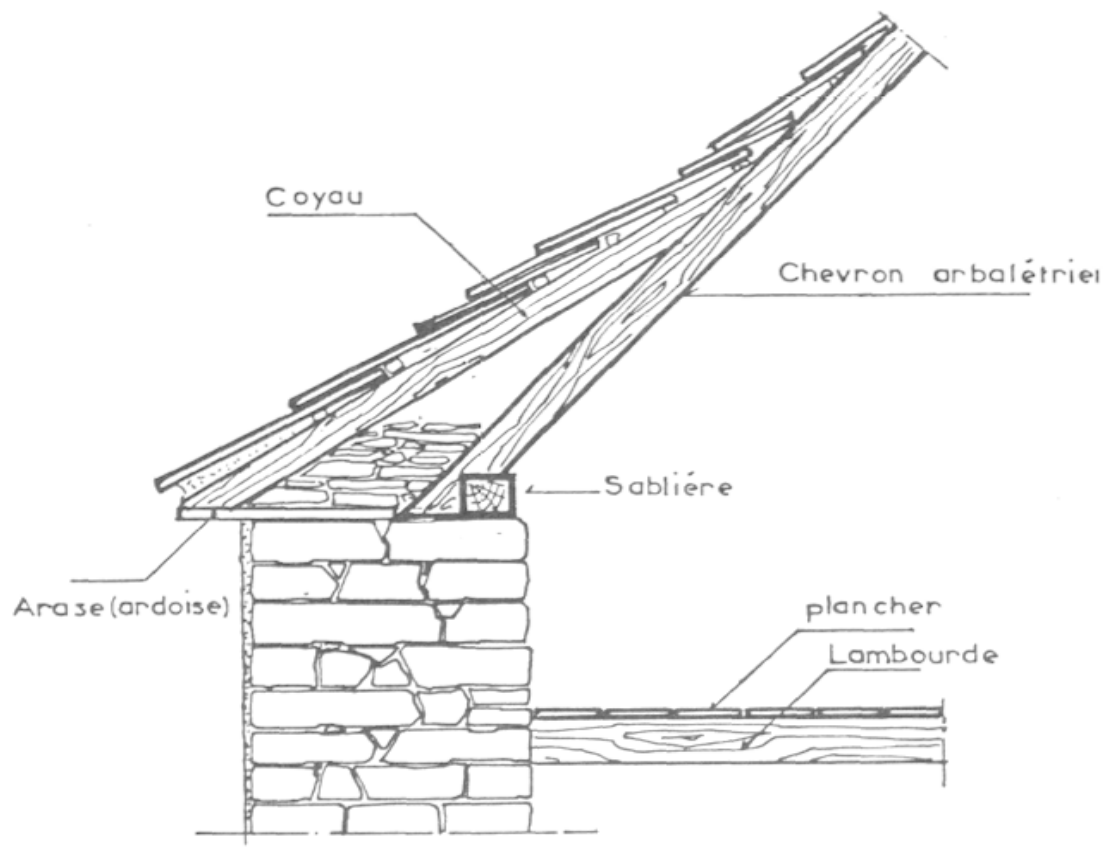

Fig. 4 - Le coyau.

C'est certainement l'élément qui permet le mieux d'illustrer la démarche de réappropriation et de réévaluation de traits de l'architecture locale. En effet, l'utilisation des toitures à fortes pentes et du coyau, en continuité avec l'architecture vernaculaire, allie la rationalité technique et fonctionnelle à l'esthétique en évitant le simple mimétisme. Cette solution est à l'opposé de la conception des bâtiments industrialisés selon un principe de juxtaposition de deux volumes, principe inadapté aux besoins des élevages locaux et en rupture totale avec les formes anciennes du bâti.

Système constructif, matériaux et savoir-faire locaux

La structure des bâtiments est constituée par une charpente en bois. Ce matériau a été choisi pour ses qualités : il est isolant, régulateur d'hygrométrie, résistant, économique. Il est aussi plus facile à mettre 
en œuvre et a un meilleur comportement au feu que le métal. Le bois utilisé est le sapin de pays. Classé en catégorie B, dévalorisé, celui-ci n'avait jamais été employé jusqu'ici pour la construction. On lui préférait, par facilité, le sapin importé du Nord, aux sections standards multiples, mais aussi beaucoup plus coûteux. Il a donc fallu concevoir une charpente et un principe constructif adaptés aux petites sections qui sont la caractéristique de ces bois locaux. Le choix de la forte pente pour couvrir le volume central est non seulement en cohérence avec les charpentes locales mais a l'avantage de rejeter plus facilement la neige, ce qui a permis d'alléger les effets de surcharge et donc de diminuer les sections de la charpente. Cette dernière est composée de portiques qui reprennent le principe classique de la charpente à entrait retroussé ${ }^{6}$. En revanche, le système d'assemblage est tout à fait contemporain : basé sur le moisage, le boulonnage et le clouage, il a non seulement l'avantage d'être très résistant mais aussi celui d'être rapide et facile à mettre en œuvre. Il est donc susceptible d'être adopté par les artisans locaux, tout en permettant la construction par les éleveurs eux-mêmes.

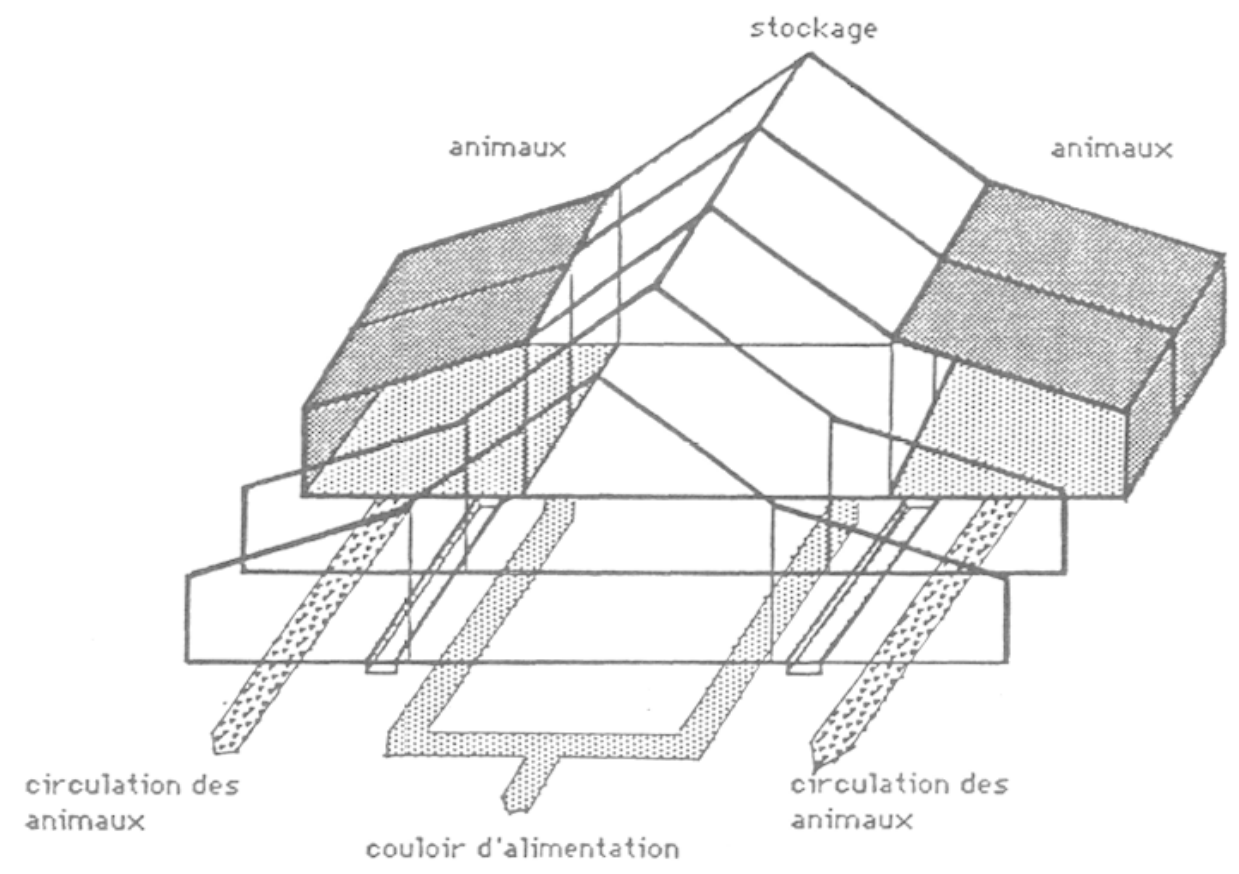

Fig. 5 - Un système évolutif.

Ce système de portiques, ancrés au sol, définit un réseau spatial modulable par addition, par soustraction ou par variation des dimensions (cf. fig. 5). Il confère donc au bâtiment un caractère évolutif et extensible

6. Charpente composée de fermes (assemblages de pièces en forme de triangle dans un plan vertical composé de deux arbalétriers, d'un poincon et d'un entrait) dont l'entrait a été placé plus haut pour dégager l'espace du comble. 
mais aussi une grande flexibilité de son espace intérieur. Il correspond aux besoins des éleveurs et des élevages locaux, extrêmement diversifiés dans leurs pratiques et leurs choix de fonctionnement, mais aussi en perpétuelle évolution.

Une fois mise en place cette structure porteuse, toutes sortes de matériaux de remplissage peuvent être utilisés. Ce sont les plus faciles à manipuler qui ont été suggérés. Les bardages de bois qui ferment les pignons ont non seulement l'avantage d'utiliser les coupes faites par les exploitants mais aussi celui de reprendre le principe de l'ancienne midiera ${ }^{7}$. Les bardages métalliques pour la couverture ont permis d'assurer au meilleur prix une adaptation aux principes volumétriques du bâtiment.

La plongée effectuée ici dans les réalités fort contradictoires que vivent les zones de montagne, préoccupées à la fois par la préservation de leur patrimoine et par la revivification économique, a permis d'esquisser quelques solutions concrètes. Solutions qui essaient d'articuler théorie et pratique en rompant avec le rapport étroit trop souvent établi entre esthétique, nature et nostalgie, mais aussi en détruisant l'idée que la modernité est en rupture avec la tradition. Cette étude fait partie des tentatives de redécouvertes des pratiques, des techniques, des savoirs et savoir-faire, des formes de vie sociale qui sont soit ignorés, soit considérés comme dépassés, dans le but de démontrer non seulement que ces pratiques continuent d'exister mais qu'elles peuvent être à l'origine de démarches de développement porteuses d'innovations à la fois techniques et sociales. Définies à l'opposé des approches conservatoires, les notions d'intégration et d'adaptation, transformées en concepts opératoires, peuvent contribuer à la relance de politiques de développement diversifiées, dans lesquelles une relation dynamique entre une architecture et son environnement prendrait un sens renouvelé.

7. Bardage : revêtement protecteur vertical, mince, en bois, métal, plastique, des murs extérieurs d'un bâtiment. Midiera : bardage en bois des pignons "sud" des greniers à foin des granges foraines, réalisé en planches non jointives. 


\section{BIBLIOGRAPHIE}

Barrué, $M$.

1985 “Architecture vernaculaire, modernité, ruralité", in colloque de l'ARF, L'habitat rural : nouveaux modèles, nouveaux usages, Amiens.

Barrué-Pastor, $M$.

1987 "Construction et protection des paysages : les ambiguïtés du développement local et de la loi montagne", Droit et Ville 24 : 217-237.

1988 "Pour une anthropologie des bâtiments d'élevage", in M. Jollivet, éd., Diversification des modèles de développement rural, Paris, L'Harmattan.

Barrué-Pastor, M. et M. Barué

1990 Architecture, élevage et société en montagne : une expérience pilote de développement local intégré dans les vallées pyrénéennes. Toulouse-Paris, Éditions du CNRS.

Barrué-Pastor, M., M. Barrué et C. Consola

1987 Bâtiments d'élevage et système social. Rapport de recherche, Toulouse, UTM-EAT-CNRS.

Béguin, $\mathbf{F}$.

1982 "Architectures et paysages", in F. Dagognet, éd. [1982] : 85-92.

Berger, P. et C. Eychenne

1987 L'enjeu esthétique de la figure architecturale. Rapport de recherche, Saint-Étienne, École d'architecture de Saint-Étienne.

Braque, $G$

1948 Cahiers 1917-19ł7. Paris, Braque Éditeur.

Briffaud, S.

1989 "La connaissance vagabonde. Les voyageurs en France et le paysage à la fin du XVIIIe siècle”, Monuments historiques 163, avr.-mai : 19-25.

Brown, R.

1989 Clefs pour une poétique de la sociologie. Arles, Actes Sud.

Chamboredon, J.-C.

1985 "La 'naturalisation' de la campagne : une autre manière de cultiver les simples", in Protection de la nature, histoire et idéologie. Paris, L'Harmattan : 138-151.

Dagognet, F., éd.

1982 Mort du paysage? Philosophie et esthétique du paysage. Paris, Éd. Champ Vallon-PUF (coll. Milieux).

Fichet, F.

1979 La théorie architecturale à l'âge classique. Paris, Éd. Pierre Mardaga. 
Giedion, S.

1978 Espace, temps, architecture. Paris, Denöel/Gonthier.

Leroi-Gourhan, A.

1945 Milieu et techniques. Paris, Albin Michel.

Moscovici, S.

1977 Essai sur l'histoire humaine de la nature. Paris, Flammarion.

Parain, C.

1979 Outils, ethnies et développement historique. Paris, Éditions Sociales.

Picon, B.

1990 "De nouvelles méthodes pour de nouveaux objets", in Pour une théorie de l'interdisciplinarité, Paris, L'Harmattan.

Schutz, A.

1954 "On Multiple Realities", Philosophy and Phenomenological Research 5 (4) : 533-576.

Segaud, $M$.

1980 Essai sur le code de l'esthétique en architecture. Paris, Institut d'Études et de Recherches architecturales (IERAU).

Venturi, R.

1976 De l'ambiguité en architecture. Paris, Dunod.

Viollet-Le-Duc

1977 Entretien sur l'architecture. Paris, Éd. Pierre Mardaga. 